\title{
Study on Crowning of helical gear shaved by CNC shaving machine with three synchronous axes
}

\author{
Ruei-Hung Hsu' ${ }^{\mathrm{a}}$, Chi-Wen Han \\ No. 100, Wenhwa Road, Seatwen, Taichung 407, Taiwan.
}

\begin{abstract}
Gear Shaving is the most efficient and economical ways to finish gears before heat treatment. The workpiece after shaving operation can reduce the running noise, which often used in the automobile industry. In this paper, we propose a mathematical model of a CNC shaving machine with multi-synchronous axes. The proposed mathematical model can be used to calculate the crowning tooth surface of the gear in parallel-shaving process.
\end{abstract}

\section{Introduction}

The gear shaving process is often used in the tooth profile finishing before gear heat treatment process. The workpiece after shaving operation can reduce the running noise, which often used in the automobile industry. In this paper, we propose a mathematical model to simulate the shaving process with crowning motion.

Litvin[1] proposed the basic mesh conditions for the crossed helical gear set. Miao and Dugas [2] develop a mathematical model of the theory plunge shaving. Seol and Litvin [3] proposed a mathematical model of the crowned helical gear after the gear shaving process. Hsu and Fong[4] proposed a mathematical model of parallel crowned shaving process with a rock mechanism. Hsu, $\mathrm{Wu}$, and Tran[5] proposed a shaving method for double crowning that has no natural twist in the tooth flanks on the work gear surfaces is proposed, which uses a variable pressure angle shaving cutter in a parallel gear shaving process.

In this paper, we derive a mathematical models for the shaving cutter and coordinate systems for the CNC shaving machine and shaving gear. We will compare the crowning in the shaving process with two and three synchronous axes $\mathrm{CNC}$ shaving machine.

\section{Basic geometry of the plunge shaving cutter}

Basic meshing conditions for the crossed helical gear set have been derived by Litvin [1] and are applied here to calculate the basic geometry data of the plunge shaving cutter. Assume the following eight data are given: teeth no. $N_{1}$, normal circular tooth thickness $s_{p n 1}$, helix angle $\beta_{p 1}$ of the shaving cutter; teeth no. $N_{2}$, normal circular tooth thickness $s_{p n 2}$, helix angle $\beta_{p 2}$ of the work gear, normal module $m_{p n}$ and pressure angle $\alpha_{p n}$ of shaving cutter and work gear. Based on Litvin's (1994) work, the following four equations are required to assure the tight meshing between involute crossed helical gears:

$$
\begin{gathered}
\gamma_{o}=\left(\beta_{o 1} \pm \beta_{o 2}\right) \\
\tan \alpha_{o n}=\tan \alpha_{o t 1} \cos \beta_{o 1}=\tan \alpha_{o t 2} \cos \beta_{o 2} \\
p_{o n}=\frac{m_{p t 1} \cos \alpha_{p t 1}}{\cos \alpha_{o t 1}} \cos \beta_{o 1}=\frac{m_{p t 2} \cos \alpha_{p t 2}}{\cos \alpha_{o t 2}} \cos \beta_{o 2} \\
N_{1} i n v \alpha_{o t 1}+N_{2} i n v \alpha_{o t 2}=N_{1}\left(\frac{s_{p t 1}}{2 r_{p 1}}+i n v \alpha_{p t 1}\right)+N_{2}\left(\frac{s_{p t 2}}{2 r_{p 2}}+i n v \alpha_{p t 2}\right)-\pi
\end{gathered}
$$

where

$$
\begin{aligned}
& m_{p t 1}=m_{n} / \cos \beta_{p 1}, \\
& m_{p t 2}=m_{n} / \cos \beta_{p 2}, \\
& \tan \alpha_{p t 1}=\tan \alpha_{p n} / \cos \beta_{p 1}, \\
& \tan \alpha_{p t 2}=\tan \alpha_{p n} / \cos \beta_{p 2}, \\
& s_{p t 1}=s_{p n 1} / \cos \beta_{p 1}, \\
& s_{p t 2}=s_{p n 2} / \cos \beta_{p 2}, \\
& r_{p 1}=m_{p t 1} N_{1} / 2,
\end{aligned}
$$$$
\text { and } \quad r_{p 2}=m_{p t 2} N_{2} / 2
$$

Eq. (1) to Eq. (4) correspond to, respectively, the helix angle, pressure angle, circular pitch, and tooth thickness compatibility derived in the normal plane. The " \pm ” sign

\footnotetext{
${ }^{a}$ Corresponding author: rhhsu@fcuoa.fcu.edu.tw
} 
in Eq. (1) indicates the same/opposite direction of helices between the shaving cutter and the work gear. The four variables, $\alpha_{o t 1}, \alpha_{o t 2}, \beta_{o 1}$, and $\beta_{o 2}$, can be solved by using Eq. (1) to Eq. (4). The operating center distance $E_{o}$ is solved by using the following equation:

$E_{o}=r_{o 1}+r_{o 2}$

where

$$
\begin{aligned}
& r_{o 1}=r_{p 1} \cos \alpha_{p t 1} / \cos \alpha_{o t 1} \\
& r_{o 2}=r_{p 2} \cos \alpha_{p t 2} / \cos \alpha_{o t 2}
\end{aligned}
$$

The start of active point (S.A.P.) and meshing point of outside radius(E.A.P.) mean the diameters of the shaving cutter meshing with the form circle and the addendum circle of the work gear respectively. The start of active point (S.A.P.) and meshing point of outside radius are as shown in the following:

$$
\begin{aligned}
& \text { S.A.P. }=\frac{2 r_{b 1}}{\cos \left(\tan ^{-1}\left(\frac{\tan \alpha_{o t 1}-r_{b 2}\left(\tan \alpha_{f 2}-\tan \alpha_{o t 2}\right) \cos \beta_{b 1}}{\cos \beta_{b 2} r_{b 1}}\right)\right)} \\
& \text { E.A.P. }=\frac{2 r_{b 1}}{\cos \left(\tan ^{-1}\left(\frac{\tan \alpha_{o t 1}-r_{b 2}\left(\tan \alpha_{a 2}-\tan \alpha_{o t 2}\right) \cos \beta_{b 1}}{\cos \beta_{b 2} r_{b 1}}\right)\right)}
\end{aligned}
$$

where

$$
\begin{aligned}
& \alpha_{a 2}=\cos ^{-1}\left(r_{b 2} / r_{a 2}\right) \\
& \alpha_{f 2}=\cos ^{-1}\left(r_{f 2} / r_{a 2}\right)
\end{aligned}
$$

\section{Mathematical model of the shaving gears coordinate systems}

The coordinate systems for gear shaving machine can be simplified as shown in Fig. 1. The coordinate system $S_{a}$ is rigidly connected to the frame of the CNC shaving machine. The coordinate systems $S_{1}$ and $S_{2}$ are rigidly connected to the shaving cutter and the gear, respectively. The parameters $\phi_{1}$ and $\phi_{2}\left(\phi_{1}\right)$ are the rotating angle of the shaving cutter and the gear, respectively. The machine movement include the traverse movement along of the axis of the gear $z_{t}$, the vertical movement crossed the axis of the gear $c z_{t}$, the center distance between the gear and the shaving cutter $E_{o}-a z_{t}^{2}$, and the crossed angle between the cutter and the gear $\gamma$.

The shaving cutter is made in the form of standard helical gear, and the mathematical model of standard helical gear has been derived by many textbooks [1]. Assume that the position vector $\mathbf{r}_{1}\left[x_{1}(u, v), y_{1}(u, v), z_{1}(u, v), 1\right]^{T}$ and the unit normal vector $\mathbf{n}_{1}\left[n_{x 1}(u, v), n_{y 1}(u, v), n_{z 1}(u, v)\right]$ of shaving cutter are given and represented in coordinate system $S_{1}$. The locus of the gear $\mathbf{r}_{2}$ represented in coordinate system $S_{2}$ is shown in Eq. (8).

$$
\mathbf{r}_{2}\left(u, v, \phi_{1}, \theta\right)=\mathbf{M}_{21}\left(\phi_{1}, z_{t}\right) \cdot \mathbf{r}_{1}(u, v)
$$

where

$$
\begin{gathered}
\mathbf{M}_{21}=\mathbf{M}_{2 d} \mathbf{M}_{d 1} \\
\mathbf{M}_{2 d}=\left[\begin{array}{cccc}
\cos \phi_{2} & \sin \phi_{2} & 0 & \cos \phi_{2}\left(\mathrm{E}_{o}-a z_{t}^{2}\right)-\sin \phi_{2} c z_{t} \\
-\sin \phi_{2} & \cos \phi_{2} & 0 & -\sin \phi_{2}\left(\mathrm{E}_{o}-a z_{t}^{2}\right)-\cos \phi_{2} c z_{t} \\
0 & 0 & 1 & z_{t} \\
0 & 0 & 0 & 1
\end{array}\right]
\end{gathered}
$$

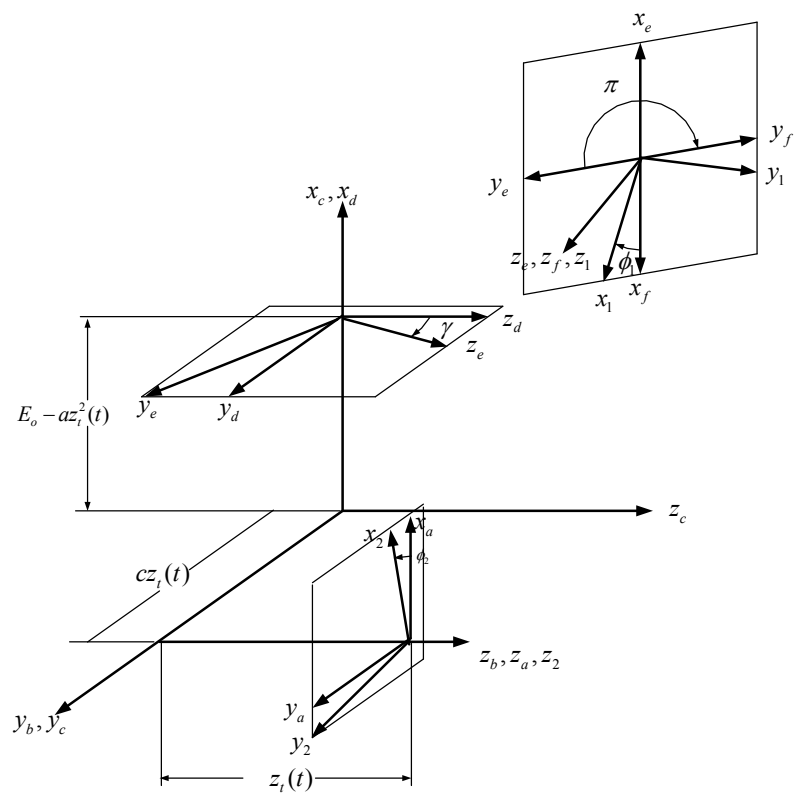

Figure 1. Coordinate systems for $\mathrm{CNC}$ shaving machine

$$
\mathbf{M}_{c 1}=\left[\begin{array}{cccc}
-\cos \phi_{1} & -\sin \phi_{1} & 0 & 0 \\
\cos \gamma \sin \phi_{1} & -\cos \gamma \cos \phi_{1} & \sin \gamma & 0 \\
-\sin \gamma \sin \phi_{1} & \cos \phi_{1} \sin \gamma & \cos \gamma & 0 \\
0 & 0 & 0 & 1
\end{array}\right]
$$

In the shaving process, the cutter is fed into the gear with table traverse in the direction of the gear longitudinal. If the gear needed to crown, the cutter is radial feed-in $a z_{t}^{2}$ along the center distance between the gear and the cutter. Therefore, the locus of the gear $\mathbf{r}_{2}$ is simplified as shown in Eq. (12):

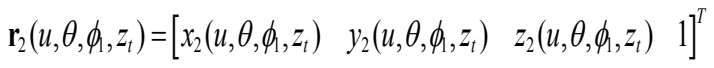

The equation of meshing between the gear and the shaving cutter is shown in Eq. (13) and (14):

$$
\begin{aligned}
& f_{1}\left(u, \theta, \phi_{1}, z_{t}\right)=\mathbf{n}_{2} \cdot \mathbf{v}_{2}^{(12)}=\frac{d \phi_{1}}{d t}\left(\frac{\partial}{\partial \phi_{1}}\left[x_{2}\left(u, \theta, \phi_{1}, z_{t}\right) \quad y_{2}\left(u, \theta, \phi_{1}, z_{t}\right) \quad z_{2}\left(u, \theta, \phi_{1}, z_{t}\right)\right]^{T}\right) \\
& f_{2}\left(u, \theta, \phi_{1}, z_{t}\right)=\mathbf{n}_{2} \cdot \mathbf{v}_{2}^{(12)}=\frac{d z_{t}}{d t}\left(\frac{\partial}{\partial z_{t}}\left[x_{2}\left(u, \theta, \phi_{1}, z_{t}\right) \quad y_{2}\left(u, \theta, \phi_{1}, z_{t}\right) \quad z_{2}\left(u, \theta, \phi_{1}, z_{t}\right)\right]^{T}\right)
\end{aligned}
$$

where

$$
\mathbf{n}_{2}=\left[\begin{array}{l}
n_{x}(u, \theta) \\
n_{y}(u, \theta) \\
n_{z}(u, \theta)
\end{array}\right]
$$


$\mathbf{L}_{12}$ is the up-submatrix of the order $(3 \times 3)$ of the matrix $\mathbf{M}_{12}$.

Gear shaving is a free cutting process that removes small amounts of metal from the working surface of gear teeth, therefore the operating pitch helical angle will be constant. According to literature the rotating angle between the shaving cutter and the gear is as shown in following equation:

$\phi_{1}=\frac{\mathrm{N}_{2}}{\mathrm{~N}_{1}} \phi_{2} \pm \frac{\tan \beta_{02}+c}{r_{o 2}} z_{2} \quad$ (+ for R.H. , - for L.H.) (16)

Where $\mathrm{N}_{1}$ and $\mathrm{N}_{2}$ are teeth number of the shaving cutter and the gear. The parallel-shaving shaved gear be solved by using Eqs. (12), (13), (14) and (16) simultaneously.

\section{Numerical examples}

The basic gear data is shown in Table 1. The basic shaving cutter setup on the CNC shaving machine, we adopt the basic meshing condition for a crossed helical gear set derived by Litvin [1]. This example mainly compares gear crowned motion for the shaving machine setting of two axes and the three synchronous axes. The shaving machine setting data is listed in table 2. The simulation crowning amount of the shaved gear of two and three synchronous axes machine as shown in Figure 2 and Figure 3, respectively. The dotted line in the topography indicates the location of the work gear's pitch radius.

Table 1. Basic data for gear, shaving cutter and machine settings.

\begin{tabular}{|c|c|}
\hline \multicolumn{2}{|c|}{ Work gear data } \\
\hline Number of teeth & 37 \\
\hline Normal module & $2.86 \mathrm{~mm}$ \\
\hline Normal circular-tooth thickness & $4.197 \mathrm{~mm}$ \\
\hline Normal pressure angle & $20^{\circ}$ \\
\hline Helix angle & $0^{\circ}$ R.H. \\
\hline Outer diameter & $110.727 \mathrm{~mm}$ \\
\hline Form diameter & $100.67 \mathrm{~mm}$ \\
\hline Face width & $16 \mathrm{~mm}$ \\
\hline \multicolumn{2}{|c|}{ Shaving cutter data } \\
\hline Number of teeth & 79 \\
\hline Helix angle & $12^{\circ}$ L.H. \\
\hline Normal circular-tooth thickness & $0.699 \mathrm{~mm}$ \\
\hline \multicolumn{2}{|l|}{ Machine settings } \\
\hline Operating center distance $\left(\mathrm{E}_{o}\right)$ & $161.584 \mathrm{~mm}$ \\
\hline Operating crossed angle $\left(\gamma_{o}\right)$ & $11.521^{\circ}$ \\
\hline
\end{tabular}

Table 2. Coefficient value of machine setting

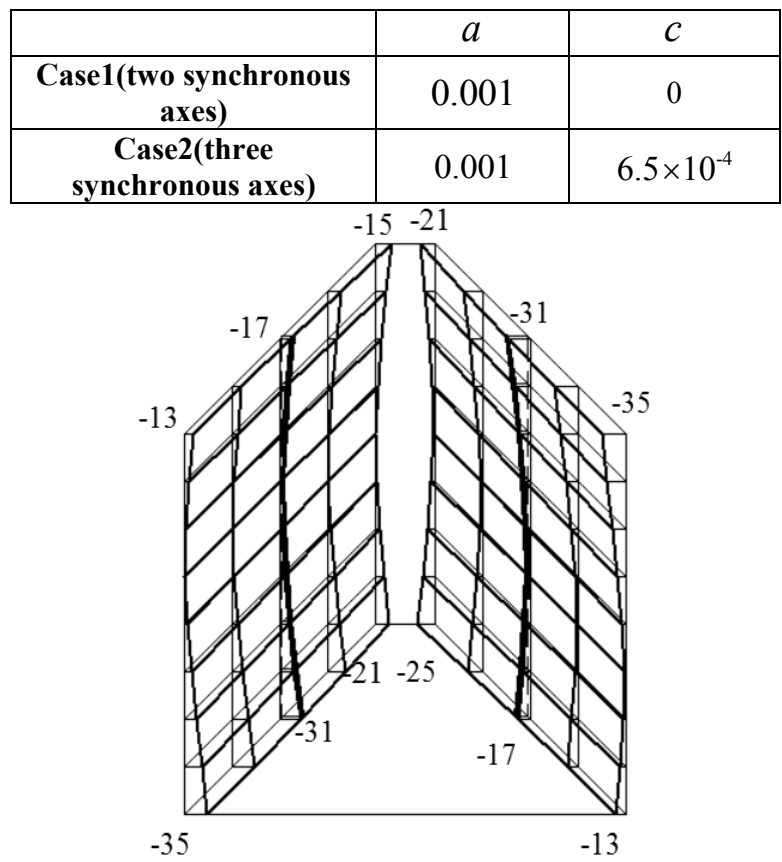

Figure 2. The topography of simulation for Case1 (two synchronous axes)

The results show that whether you use a two-axis or three-axis synchronous shaving machine, the gear tooth surface will be twist. However, using a three-axis synchronous shaving machine will not cause twist in the pitch circle of the gear.

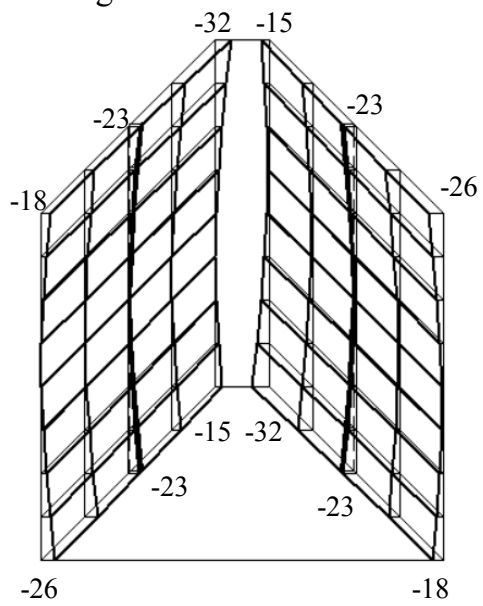

Figure 3. The topography of simulation for Case2 (three synchronous axes)

\section{Acknowledgments}

The authors are grateful to the National Science Council of the R.O.C for the financial support. Part of this work was performed under Contract No. 107-2221-E-035 -062.

\section{References}

1. Litvin, F.L., Gear Geometry and Applied Theory, PTR Prentice Hall, Englewood Cliffs, NJ, (1994) 412-468 
2. Miao, H.C. Koga, H., Design and analysis of plunge shaving for finishing gears with tooth profile modifications, ASME, Power Transmission and Gearing Conference, San Diego, CA, 88 (1996).

3. Seol, I.H., Litvin, F.L., Computerized generation and simulation of meshing of modified spur and helical gears manufactured by shaving, Computer Methods in Applied Mechanics and Engineering 190 (39) (2001) 5037-5055.
4. Ruei-Hung Hsu, and Zhang-Hua Fong, 2010,"An Auxiliary Crowning Analysis of Parallel Gear Shaving." Mechanism and Machine Theory 45 ,pp. 1298-1313.

5. Ruei-Hung Hsu, Wu Yu-Ren and Van-The Tran, 2017 Manufacturing helical gears with doublecrowning and twist-free tooth flanks using a variable pressure angle shaving cutter (Proceedings of the Institution of Mechanical Engineers, Part B: Journal of Engineering Manufacture) pp 1-10 